\title{
Photodissociation of organic molecules in star-forming regions
}

\section{Methanol}

\author{
S. Pilling ${ }^{1}$, R. Neves ${ }^{2}$, A. C. F. Santos ${ }^{3}$, and H. M. Boechat-Roberty ${ }^{2}$ \\ 1 Laboratório Nacional de Luz Síncrotron, Caixa Postal 6192, CEP 13084-971, Campinas, SP, Brazil \\ e-mail: spilling@lnls.br \\ 2 Observatório do Valongo, Universidade Federal do Rio de Janeiro, Ladeira Pedro Antônio 43, CEP 20080-090, \\ Rio de Janeiro, RJ, Brazil \\ 3 Instituto de Física, Universidade Federal do Rio de Janeiro, Caixa Postal 68528, CEP 21941-972, Rio de Janeiro, \\ RJ, Brazil
}

Received 18 August 2006 / Accepted 11 December 2006

\begin{abstract}
The presence of methyl alcohol or methanol $\left(\mathrm{CH}_{3} \mathrm{OH}\right)$ in several astrophysical environments has been characterized by its high abundance that depends on both the production rate and the destruction rate. In the present work, the photoionization and photodissociation processes of methanol have been experimentally studied, employing soft X-ray photons (100-310 eV) from a toroidal grating monochromator (TGM) beamline of the Brazilian Synchrotron Light Laboratory (LNLS). Mass spectra were obtained using the photoelectron photoion coincidence (PEPICO) method. Kinetic energy distribution and abundances for each ionic fragment have been obtained from the analysis of the corresponding peak shapes in the mass spectra. Absolute photoionization and photodissociation cross sections were also determined. We have found, among the channels leading to ionization, about $11-16 \%$ of $\mathrm{CH}_{3} \mathrm{OH}$ survive the soft X-rays photons. This behavior, together with an efficient formation pathways, may be associated with the high column density observed in star-forming regions. The three main photodissociation pathways are represented by $\mathrm{COH}^{+}\left(\right.$or $\mathrm{HCO}^{+}$) ion release (with ejection of $\mathrm{H}_{2}+\mathrm{H}$ ), the dissociation via $\mathrm{C}-\mathrm{O}$ bond rupture (with strong charge retention preferentially on the methyl fragment) and the ejection of a single energetic $(2-4 \mathrm{eV})$ proton. Since methanol is very abundant in star forming regions, the produced protons could be an alternative route to molecular hydrogenation or a trigger for secondary dissociation processes or even to promote extra heating of the environment.
\end{abstract}

Key words. astrochemistry - methods: laboratory - ISM: molecules - X-rays: ISM - molecular data - molecular processes

\section{Introduction}

Methyl alcohol or methanol $\left(\mathrm{CH}_{3} \mathrm{OH}\right)$, the simplest alcohol, is one of the most abundant molecules detected toward various astrophysical regions, including hot molecular cores (HMCs) associated with low- and high-mass star-forming regions (Requena-Torres et al. 2006; Remijan et al. 2004; Pei et al. 2000; De Buizer et al. 2000; Menten 1991; Norris et al. 1993; Caswell et al. 1993, and references therein); dense molecular clouds (Irvine et al. 1987; Tielens \& Alamandola 1987), circumstellar and proto-stellar regions (Schutte et al. 1999; Keane et al. 2001; Goldsmith et al. 1999; Pontoppidan et al. 2003, 2004; Grim et al. 1991) and in comets (Mumma et al. 2001; BockeleeMorvan et al. 1994; Crovisier \& Bockelée-Morvan 1999, and references therein). In these objects, the radiation field can drive several photophysical and photochemical processes, including molecular photodissociation. The products of organic molecule dissociation (e.g. reactive ions and radicals) can fuel the formation of interstellar complex molecules such as long carbon chain molecules.

The maser emission of $\mathrm{CH}_{3} \mathrm{OH}$ in the $\mathrm{GHz}$ range is its main fingerprint and, to date, at least six catalogues have presented this spectroscopic signature in star forming regions (e.g. Schutte et al. 1993; Caswell et al. 1995; Walsh et al. 1997; Slysh et al. 1999; Szymczak et al. 2000). The X-ray photons from star-forming regions are capable of traversing large column densities of gas before being absorbed. X-ray-dominated regions (XDRs) in the interface between the ionized gas and the self-shielded neutral layers could influence the selective heating of the molecular gas. The complexity of these regions possibly allows a combination of different scenarios and excitation mechanisms to coexist together (Goicoechea et al. 2004).

The formation mechanisms for interstellar methanol occur both in the gas phase and on grain surfaces. In dense clouds, methanol is frozen onto grains. The enhanced abundance of methanol in the warm gas has been taken as evidence that the methanol is released from grains in these regions.

Both the UMIST (Millar et al. 1991) and Standard Model (Lee et al. 1996) reaction data set include a particularly simple gas-phase chemistry for methanol formation directly via radiative associative reaction of a methyl radical and water molecules followed by electronic recombination with hydrogen liberation

$\mathrm{CH}_{3}+\mathrm{H}_{2} \mathrm{O} \longrightarrow \mathrm{CH}_{3} \mathrm{OH}_{2}^{+}+h v \stackrel{\mathrm{e}^{-}}{\longrightarrow} \mathrm{CH}_{3} \mathrm{OH}+\mathrm{H}$.

However, as pointed by Millar et al. (1991) the gas phase reactions would not be enough to justify the relative methanol abundances detected, of about $10^{-7}$ or more, and grain mantle involvement was required. 
From the theoretical models of Tielens \& Whittet (1997) and Charnley et al. (1997) the successive hydrogenation of CO in interstellar ices

$$
\mathrm{CO} \stackrel{\mathrm{H}}{\longrightarrow} \mathrm{HCO} \stackrel{\mathrm{H}}{\longrightarrow} \mathrm{H}_{2} \mathrm{CO} \stackrel{\mathrm{H}}{\longrightarrow} \mathrm{CH}_{3} \mathrm{O} \text { and/or }
$$

$\mathrm{CH}_{2} \mathrm{OH} \stackrel{\mathrm{H}}{\longrightarrow} \mathrm{CH}_{3} \mathrm{OH}$

has been proposed to produce molecules like methanol. Sorrel (2001) has also proposed that accretion of gas-phase $\mathrm{H}, \mathrm{O}, \mathrm{OH}$, $\mathrm{H}_{2} \mathrm{O}, \mathrm{CH}_{4}, \mathrm{NH}_{3}$ and $\mathrm{CO}$ onto dust grains sets up a carbonoxygen-nitrogen chemistry in the grain that can also produce methanol. As a consequence, a high concentration of free $\mathrm{OH}$, $\mathrm{CH}_{3}$ and $\mathrm{NH}_{2}$ radicals is created in the grain mantle mainly by photolysis reactions. Once these radicals are created, they remain frozen in position until the grains heat up. As this occurs, the radicals become mobile and undergo chemical reactions among themselves and with other adsorbed molecules to produce complex organic molecules including $\mathrm{CH}_{3} \mathrm{OH}$.

Over the years, many experimental studies involving UV photolysis and proton bombardment on ices were tested and some of these routes have been fully accepted, Shalabiea \& Greemberg (1994) have proposed a route for formation of solid methanol on grain mantles from UV photolysis of formaldehyde-water ices,

$\mathrm{H}_{2} \mathrm{CO}+\mathrm{H}_{2} \mathrm{O}+h v \longrightarrow \mathrm{CH}_{3} \mathrm{OH}+\mathrm{O}$.

They predict a methanol photoproduction rate per incident photon of about $1.6 \times 10^{-3}$ photon $^{-1}$.

On the other hand, Allamandola et al. (1988) and Shuttle et al. (1996) have performed UV photolysis of $\mathrm{H}_{2} \mathrm{O}+\mathrm{CO}$ ice but the methanol yields are insufficient to explain the observed abundances in interstellar ices.

Another set of reaction pathways was studied by Moore \& Hudson (1998) and Hudson \& Moore (1999) who employed proton bombardment on $\mathrm{CO}+\mathrm{H}_{2} \mathrm{O}$ and $\mathrm{CH}_{4}+\mathrm{H}_{2} \mathrm{O}$ ices,

$\mathrm{CO}+\mathrm{H}_{2} \mathrm{O} \stackrel{\text { protons }}{\longrightarrow} \mathrm{CH}_{3} \mathrm{OH}+\mathrm{O}$

and

$\mathrm{CH}_{4}+\mathrm{H}_{2} \mathrm{O} \stackrel{\text { protons }}{\longrightarrow} \mathrm{CH}_{3} \mathrm{OH}+\mathrm{H}_{2}$.

The results have shown that the methanol yield on both carbon monoxide-water and methane-water ices, despite the evident production of methanol, could not justify alone the detected $\mathrm{CH}_{3} \mathrm{OH}$ abundance in interstellar ices. However, perhaps due to the high abundance of interstellar carbon monoxide-water ices, this process may dominates the interstellar methanol production. In summary, some of the above mentioned methanol formation routes seem able to account for the observed abundances.

Watanabe \& Kouchi (2002), also using hydrogen bombardment on $\mathrm{CO}+\mathrm{H}_{2} \mathrm{O}$ ices, have found a methanol yield up to $17 \%$ for a proton dose of about $4 \times 10^{18} \mathrm{H}^{+} / \mathrm{cm}^{2}$, revealing a large correlation of methanol production with the proton flux.

The photodissociation of methanol in the gas phase has been studied experimentally and theoretically in the ultraviolet region by several authors (Shi et al. 2002; Tang et al. 2002; Zavilopulo 2005). However, despite some photoabsorption studies in the X-ray range (Ishii \& Hitchcook 1988; Prince et al. 2003; Burton et al. 1992) there are few studies focusing on the photodissociation pathways in this photon energy range. Azuma et al. (2005) have investigated the state-selective dissociation processes of the O1s core-excited methanol and deuterated methanol.
Stolte et al. (2002) were able to experimentally isolate a specific bond-breaking process in methanol $\left(\mathrm{CH}_{3} \mathrm{OH}\right)$ by observing the anion fragment, $\mathrm{OH}^{-}$.

The present work aims to examine the photoionization and photodissociation of gaseous methanol by soft X-rays, from $100 \mathrm{eV}$ up to $310 \mathrm{eV}$, including the energies around the carbon $\mathrm{K}$ edge (C1s edge) at $\sim 290 \mathrm{eV}$. In Sect. 2, we present briefly the experimental setup. The results of the photoionization and photodissociation of the methanol molecule in the presence of soft $\mathrm{X}$-ray photons, together with the main photodissociation pathways and the determination of the absolute cross sections are presented and discussed in Sect. 3. Finally, in Sect. 4, final remarks and conclusions are given.

\section{Experimental}

The experiment was performed at the Brazilian Synchrotron Light Laboratory (LNLS), in Campinas, São Paulo, Brazil. The experimental setup has been described in detail by (Boechat-Roberty et al. 2005 - Paper I; Pilling, et al. 2006 Paper II). Briefly, soft X-ray photons in the energy range of 100 to $310 \mathrm{eV}$, from a toroidal grating monochromator (TGM) beamline, perpendicularly intersect the effusive gaseous sample inside a high vacuum chamber. The ionized (cation) recoil fragments produced by the interaction with the radiation were accelerated and detected by two micro-channel plate detectors, after massto-charge $(\mathrm{m} / \mathrm{q})$ analyzing by a time-of-flight mass spectrometer. Conventional time-of-flight mass spectra (TOF-MS) were obtained using the correlation between one Photoelectron and a Photoion Coincidence, PEPICO, described by Santos et al. (2001), Boechat-Roberty et al. (2005) and Pilling et al. (2006). Negative ions may also be produced and detected, but the corresponding cross-sections are negligible.

The base pressure in the vacuum chamber was in the $10^{-8}$ Torr range. During the experiment the chamber pressure was maintained below $10^{-5}$ Torr. The pressure at the interaction region (volume defined by the gas beam and the photon beam intersection) was estimated to be $\sim 1$ Torr $\left(10^{16} \mathrm{mols} \mathrm{cm}^{-3}\right)$. The measurements were done at room temperature. The methanol sample was commercially obtained from Sigma-Aldrich with purity better than $99.5 \%$. No further purification was used except for degassing the liquid sample by multiple freeze-pumpthaw cycles before admitting the vapor into the chamber.

\section{Results and discussion}

Figure 1 shows the mass spectrum of methanol obtained at the photon energy of $288 \mathrm{eV}$, over the $\mathrm{C} 1 \mathrm{~s} \rightarrow 3 \mathrm{~s}$ resonance energy (Prince et al. 2003). We can see, for example, the methyl group fragments (mass from 12 to $15 \mathrm{amu}$ ) and the CO group (28 to $31 \mathrm{amu}$ ). The $\mathrm{O}$ group and the recombination water ion (16 to $18 \mathrm{amu}$ ) are also produced. There are also small a mounts of reactive $\mathrm{H}_{2}$ and $\mathrm{H}_{3}$ ions. The photodissociation pathways for these species become more effective in multiple ionization processes. These features will be described in detail in a future publication together with the same behavior in other highly hydrogenated organic molecules. The parent ion $\mathrm{CH}_{3} \mathrm{OH}^{+}$peak remains with high intensity showing the intrinsic stability of methanol at this photon energy.

The most produced ions are $\mathrm{COH}^{+}\left(\right.$or $\left.\mathrm{HCO}^{+}\right), \mathrm{CH}_{2} \mathrm{OH}^{+}$, and $\mathrm{H}^{+}$, followed by the parent ion and the methyl group. 


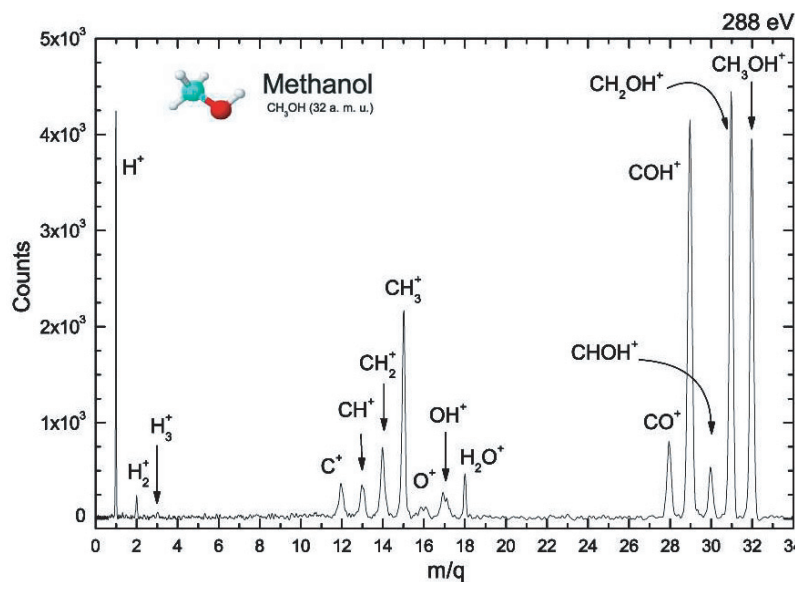

Fig. 1. Time-of-flight mass spectrum of methanol after exposure to $288.3 \mathrm{eV}$ X-ray.

\subsection{Partial ion yield and kinetic energy release}

The partial ion yield (PIY), or relative intensities, for each fragment $i$ were obtained by

$P I Y_{i}=\left(\frac{A_{i}}{A_{\mathrm{t}}^{+}} \pm \frac{\sqrt{A_{i}}+A_{i} \times E R / 100}{A_{\mathrm{t}}^{+}}\right) \times 100 \%$

where $A_{i}$ is the area of a fragment peak, $A_{\mathrm{t}}^{+}$is the total area of the PEPICO spectrum. The error factor $E R(2 \%)$ is the estimated error factor due to the data treatment.

Figures $2 \mathrm{a}-\mathrm{c}$ show the relative intensities (PIY) of fragments from methanol photodissociation in the photon energies range of 100 to $310 \mathrm{eV}$. Figure $2 \mathrm{a}$ presents the $\mathrm{CO}$ group fragments and also the parent ion $\mathrm{CH}_{3} \mathrm{OH}^{+}$, in Figs. $2 \mathrm{~b}$ and $2 \mathrm{c}$, we can see the $\mathrm{C}$ group fragments and $\mathrm{O}$ group fragments, respectively. In the bottom figure we could also see the PIY of $\mathrm{H}^{+}, \mathrm{H}_{2}^{+}$and $\mathrm{H}_{3}^{+}$. The location of the ionization potential (IP) at $292.32 \mathrm{eV}$ and the $\mathrm{C} 1 \mathrm{~s} \rightarrow 3 \mathrm{~s}$ resonance (Prince et al. 2003) are indicated in the figures. The statistical uncertainties were below $10 \%$.

A clear bump can be seen in the fractions of all released fragments near the $\mathrm{C} 1 \mathrm{~s}$ resonances and the IP energies. The methyl ion as a result of the neutral $\mathrm{OH}$ release represent about 8 to $10 \%$ of the total ion yield. Its counterpart, the hydroxyl ion, as a result of the neutral fragments liberation, $\mathrm{CH}_{3}\left(\right.$ or $\left.\mathrm{CH}_{2}+\mathrm{H}\right)$, represent only about 3 to $4 \%$ of the total ion yield. This large difference between the yield of these two fragments, after $\mathrm{C}-\mathrm{O}$ bond rupture indicates that, during the dissociation process, there is a significant preference in the charge retention by methyl over the hydroxyl. This behavior has also been seen in the photodissociation of acetic acid (Paper II).

We present in Fig. 3, a comparison between partial ion yield of the $\mathrm{CH}_{3} \mathrm{OH}$ fragments by soft X-rays $(288 \mathrm{eV})$ and the fragment yield upon bombardment with $70 \mathrm{eV}$ electrons measured at National Institute of Standards and Technology (NIST). The dissociation induced by $70 \mathrm{eV}$ electrons is very similar to the dissociation induced by $21.21 \mathrm{eV}$ (He I Lamp) photons. In both cases the ionization occurs in the valence shell. The degree of destruction of the methanol is at least twice as large in the soft $\mathrm{X}$-ray case than by photons in the UV region. Several fragments present a different dissociation pattern, as far as PIY are concerned, in X-ray field when compared to the UV field. As an example, we mention the large enhancement of $\mathrm{CH}_{2} \mathrm{OH}^{+}$produced by UV radiation with respect to the opposite behavior presented by $\mathrm{CH}_{3}^{+}$and all lower mass ions which seem to be more efficiently produced by X-rays photons. The $\mathrm{COH}^{+}\left(\right.$or $\left.\mathrm{HCO}^{+}\right)$
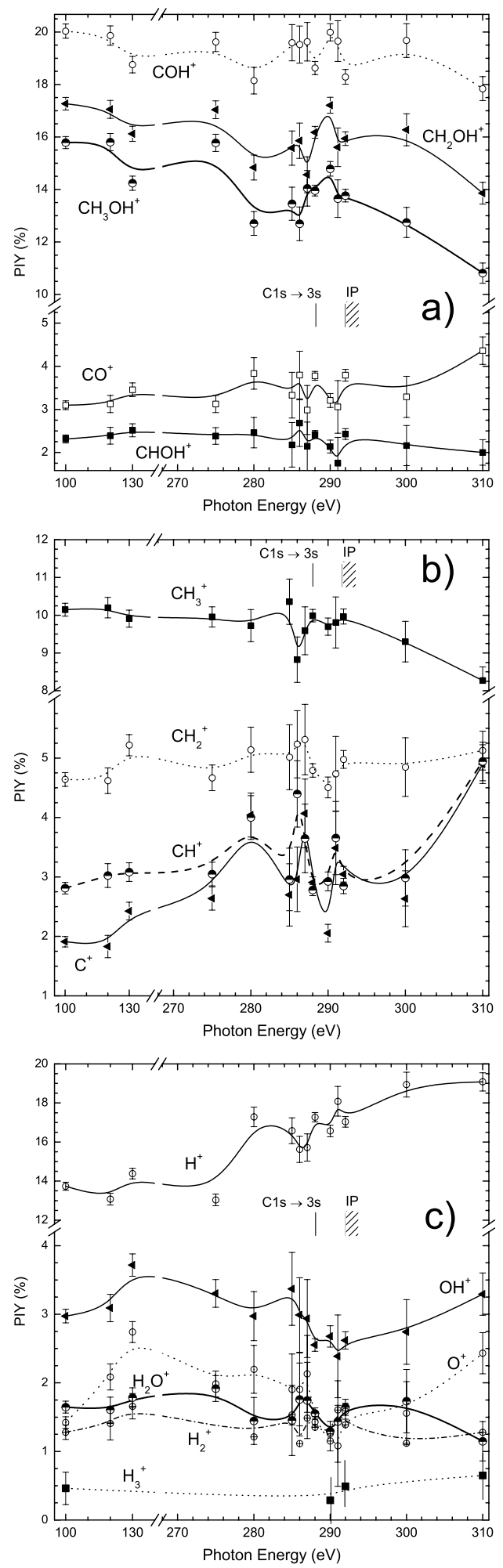

Fig. 2. Partial ion yield (PIY) of the fragments release by the $\mathrm{CH}_{3} \mathrm{OH}$ molecule as a function of photon energy. See details in text.

and $\mathrm{CHOH}^{+}$(or $\mathrm{H}_{2} \mathrm{CO}^{+}$) ion yields seem to present only small changes with respect to photon energies in the UV-X-ray range.

The absence of more doubly ionized fragments in the PEPICO spectra indicates that doubly ionized methanol dissociates preferentially via charge separation. The dynamics of the doubly and triple ionized methanol molecule will be the subject of study of a future publication. 


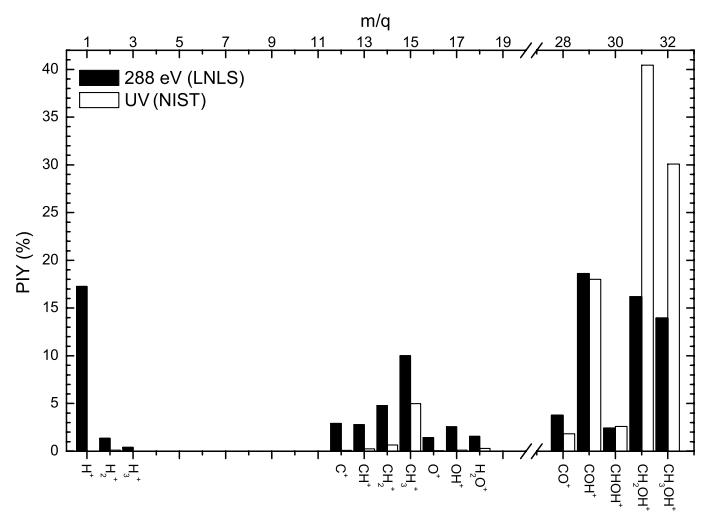

Fig. 3. Comparison between partial ion yield (PIY) of methanol fragments in soft X-ray and $70 \mathrm{eV}$ electrons measured at (NIST). The dissociation induced by $70 \mathrm{eV}$ electrons is very similar to the dissociation induced by $21.21 \mathrm{eV}$ UV photons.

As pointed out by several authors (Largo 2004; Woon 2002, and references therein) understanding the pathways of formation of biomolecules present in star-forming regions and other gaseous-dusty astronomical media is extremely important not only to classify the chemistry of those regions and, but also, to give some clues about the origin and possible spread of life in the Universe. Despite the success of ab initio theoretical calculations, the endothermic ion-molecule reactions are not considered as viable in the interstellar medium and only exothermic reactions have been accepted as viable mechanisms. However, with the knowledge of the kinetic energy (or at least with its value range) of some radical and ionic photofragments, some endothermic ion-molecule reactions could be competitive and, in extreme situations, even become more efficient than those exothermic reactions.

We have determined the kinetic energy of all cationic fragments from the X-ray photodissociation of methanol. The present time-of flight spectrometer was designed to fulfil the Wiley-McLaren conditions for space focusing (Wiley \& McLaren 1955). Within the space focusing conditions, the observed broadening of peaks in spectra is mainly due to the spread in kinetic energy of the fragments released. Considering that the electric field in the interaction region is uniform, we can determine the released energy in the fragmentation process $\left(U_{0}\right)$ from each peak width, with the formula used by Hansen et al. (1998) and Santos et al. (2001)

$U_{0}=\left(\frac{q E \Delta t}{2}\right)^{2} \frac{1}{2 m}$

where $q$ is the ion fragment charge, $E$ the electric field in the interaction region, $m$ is the mass of the fragment, and $\Delta t$ is the time peak width $(F W H M)$ taken from PEPICO spectra. In order to test the above equation we have measured the argon mass spectrum under the same conditions.

The calculated values for kinetic energy release $\left(U_{0}\right)$ for methanol fragmentation are shown in Table 1 . We observe that the highest kinetic energy release (up to $3.8 \mathrm{eV}$ ) was associated with the lightest fragment $\mathrm{H}^{+}(m / q=1)$ followed by $\mathrm{H}_{2}^{+}$ $(m / q=2), \mathrm{H}_{3}^{+}(m / q=3)$ and other ions like $\mathrm{C}^{+}$and $\mathrm{O}^{+}$, as expected. In our previous formic acid photodissociation results (Paper I), we found extremely fast ionic fragments $\left(U_{0}>10 \mathrm{eV}\right)$ that were associated with dissociation of doubly or multiplycharged ions at high photon energies.

The surface potentials of the ionic states are extremely repulsive. For core excited molecules which dissociate into one charged and one or more neutral fragments, the dissociation is primarily controlled by chemical (non-Coulomb) forces originating from the residual valence electrons of the system (Nenner \& Morin 1996). From Table 1, one can see that the mean kinetic energy release, $U_{0}$, of some methanol fragments increases as the photon energy approximates the C 1 s edge (288-292 eV). This enhancement is due to the repulsive character of the $\sigma^{*}\left(\pi^{*}\right)$ resonance.

\subsection{Photodissociation and formation pathways}

The present work shows the photofragmentation of methanol produced by soft X-rays and compares the yields of these fragments with those produced by $70 \mathrm{eV}$ electrons. The inner shell photoionization process may produce instabilities on molecular structure (nuclear rearrangements) leading them to peculiar dissociation pathways. From our data we could determine the main photodissociation pathways from single ionization over the $\mathrm{C} 1 \mathrm{~s}$ photon energy range. These photodissociation pathways are shown in Table 2 for $288 \mathrm{eV}$. Only events greater than $2 \%$ were considered here. The main dissociation leads to production of $\mathrm{COH}^{+}+\mathrm{H}_{2}+\mathrm{H}(\sim 19 \%)$. However the single $\mathrm{C}-\mathrm{H}$ bond rupture as a result of both $\mathrm{H}^{+}+$neutrals fragments or $\mathrm{H}_{2} \mathrm{COH}^{+}+\mathrm{H}$, represent a combined yield of about $34 \%$ of the photodissociation channels. The fragments released due to the $\mathrm{C}-\mathrm{O}$ bond rupture also represent an important route of photodissociation $(\sim 12 \%)$, for example the $\mathrm{CH}_{3}^{+}+\mathrm{OH}$ and $\mathrm{CH}_{3}+\mathrm{OH}^{+}$product routes.

Herbst \& Leung (1986) have presented several pathways for the synthesis of complex molecules in dense interstellar clouds via gas-phase chemistry models. The authors presented a significant amount of normal ion-molecule reactions including the ions $\mathrm{C}^{+}, \mathrm{CH}^{+}, \mathrm{OH}^{+}, \mathrm{CO}^{+}, \mathrm{CH}_{2}^{+}, \mathrm{H}_{2} \mathrm{O}^{+}, \mathrm{HCO}^{+}, \mathrm{CH}_{3}^{+}$, etc. In another set of reactions they have shown several radiative association reactions including the ions $\mathrm{C}^{+}, \mathrm{CH}_{3}^{+}, \mathrm{HCO}^{+}$, could lead to the production of high molecular complexity species. This work points out the importance of the ionic species in the increase of interstellar molecular complexity.

As a consequence of the high production of $\mathrm{COH}^{+}$(or $\mathrm{HCO}^{+}$) from methanol photodissociation by soft X-rays, we expect that a fraction of the detected $\mathrm{COH}^{+}\left(\right.$or $\left.\mathrm{HCO}^{+}\right)$in the interstellar medium (mainly at XDRs and HMCs) comes from methanol, since $\mathrm{CH}_{3} \mathrm{OH}$ is one of the mostly abundant molecule in these regions.

The work presented here strongly suggests that a great number of ions could be produced by the X-ray photodissociation of large organic molecules in star forming regions. Therefore, the knowledge of the photodissociation processes and its ions yields has an essential role in the interstellar chemistry scenario.

\subsection{Absolute photoionization and photodissociation cross-sections}

The absolute cross section values for both photoionization $\left(\sigma_{\mathrm{ph}-\mathrm{i}}\right)$ and photodissociation $\left(\sigma_{\mathrm{ph}-\mathrm{d}}\right)$ of organic molecules are extremely important as input for molecular abundances models. Sorrell (2001) has presented a theoretical model in which biomolecules are formed inside the bulk of icy grain mantles photoprocessed by starlight (ultraviolet and soft X-rays photons). However, the main uncertainty of this equilibrium abundance model comes from the uncertainty of the $\sigma_{\mathrm{ph}-\mathrm{d}}$ value. Therefore the precise determination of $\sigma_{\mathrm{ph}-\mathrm{d}}$ of biomolecules is very important to properly estimate the molecular abundance of those molecules in the interstellar medium that have been 
Table 1. Relative intensities (PIY) and kinetic energy $U_{0}$ release by fragments in the methanol mass spectra, as a function of photon energy (at $100,288,292$ and $310 \mathrm{eV}$ ). Only fragments with intensity $>0.1 \%$ were tabulated. The estimated experimental error was below $10 \%$.

\begin{tabular}{lllrrrr}
\hline \hline & Fragments & & \multicolumn{3}{c}{ PIY $(\%) / U_{0}(\mathrm{eV})$} \\
\cline { 1 - 2 } \cline { 5 - 6 }$m / q$ & Attribution & $100 \mathrm{eV}$ & $288 \mathrm{eV}$ & $292 \mathrm{eV}$ & $310 \mathrm{eV}$ \\
\hline 1 & $\mathrm{H}^{+}$ & $13.7 / 2.2$ & $17.3 / 2.9$ & $17.0 / 3.0$ & $19.1 / 3.8$ \\
2 & $\mathrm{H}_{2}^{+}$ & $1.3 / 1.5$ & $1.3 / 3.0$ & $1.4 / 2.9$ & $1.3 / 1.1$ \\
3 & $\mathrm{H}_{3}^{+}$ & $0.5 / 0.98$ & $0.4 / 2.4$ & $0.5 / 2.0$ & $0.6 / 0.18$ \\
12 & $\mathrm{C}^{+}$ & $1.9 / 0.61$ & $2.9 / 0.98$ & $3.0 / 0.70$ & $4.9 / 0.50$ \\
13 & $\mathrm{CH}^{+}$ & $2.8 / 0.37$ & $2.8 / 0.78$ & $2.8 / 0.78$ & $4.9 / 0.78$ \\
14 & $\mathrm{CH}_{2}^{+}$ & $4.6 / 0.43$ & $4.8 / 0.35$ & $4.9 / 0.35$ & $5.1 / 0.34$ \\
15 & $\mathrm{CH}_{3}^{+}$ & $10.1 / 0.14$ & $9.9 / 0.14$ & $9.9 / 0.15$ & $8.3 / 0.19$ \\
16 & $\mathrm{O}^{+}$ & $1.4 / 1.2$ & $1.4 / 2.2$ & $1.6 / 1.59$ & $2.4 / 1.1$ \\
17 & $\mathrm{OH}^{+}$ & $2.9 / 0.69$ & $2.2 / 0.28$ & $2.6 / 0.98$ & $3.3 / 1.7$ \\
18 & $\mathrm{H}_{2} \mathrm{O}^{+}$ & $1.6 / 0.05$ & $1.5 / 0.03$ & $1.6 / 0.04$ & $1.1 / 0.12$ \\
28 & $\mathrm{CO}^{+}$ & $3.1 / 0.14$ & $3.8 / 0.05$ & $3.8 / 0.10$ & $4.4 / 0.21$ \\
29 & $\mathrm{COH}^{+}$or $\mathrm{HCO}^{+}$ & $20.0 / 0.13$ & $18.6 / 0.10$ & $18.3 / 0.11$ & $17.9 / 0.13$ \\
30 & $\mathrm{HCOH}^{+}$or $\mathrm{H}_{2} \mathrm{CO}^{+}$ & $2.3 / 0.05$ & $2.4 / 0.07$ & $2.4 / 0.07$ & $2.0 / 0.07$ \\
31 & $\mathrm{H}_{2} \mathrm{COH}^{+}$ & $17.3 / 0.04$ & $16.2 / 0.05$ & $15.9 / 0.06$ & $13.8 / 0.07$ \\
32 & $\mathrm{CH}_{3} \mathrm{OH}^{+}$ & $15.8 / 0.03$ & $13.9 / 0.03$ & $13.8 / 0.03$ & $10.8 / 0.03$ \\
\hline
\end{tabular}

Table 2. Main photodissociation pathways from single ionization due to soft X-ray photons $(288 \mathrm{eV})$.

\begin{tabular}{lll}
\hline \hline $\mathrm{CH}_{3} \mathrm{OH}+h v$ & $\longrightarrow$ & $\mathrm{CH}_{3} \mathrm{OH}^{+}+\mathrm{e}^{-}$ \\
\hline $\mathrm{CH}_{3} \mathrm{OH}^{+}$ & $\stackrel{18.6 \%}{\longrightarrow}$ & $\mathrm{COH}^{+}+3 \mathrm{H}\left(\right.$ or $\left.\mathrm{H}_{2}+\mathrm{H}\right)$ \\
& $\stackrel{17.3 \%}{\longrightarrow}$ & $\mathrm{H}^{+}+$neutrals \\
& $\stackrel{16.2 \%}{\longrightarrow}$ & $\mathrm{H}_{2} \mathrm{COH}^{+}+\mathrm{H}$ \\
& $\stackrel{9.9 \%}{\longrightarrow}$ & $\mathrm{CH}_{3}^{+}+\mathrm{OH}\left(\right.$ or $\left.\mathrm{O}_{2}+\mathrm{H}\right)$ \\
& $\stackrel{4.8 \%}{\longrightarrow}$ & $\mathrm{CH}_{2}^{+}+\mathrm{H}_{2} \mathrm{O}\left(\right.$ or $\left.\mathrm{OH}_{2}+\mathrm{H}\right)$ \\
& $\stackrel{3.8 \%}{\longrightarrow}$ & $\mathrm{CO}^{+}+$neutrals \\
& $\stackrel{2.8 \%}{\longrightarrow}$ & $\mathrm{CH}^{+}+$neutrals \\
& $\stackrel{2.9 \%}{\longrightarrow}$ & $\mathrm{C}^{+}+$neutrals \\
& $\stackrel{2.4 \%}{\longrightarrow}$ & $\mathrm{HCOH}^{+}+\mathrm{H}_{2}($ or $\mathrm{H}+\mathrm{H})$ \\
& $\mathrm{OH}^{+}+\mathrm{CH}_{3}\left(\right.$ or $\left.\mathrm{CH}_{2}+\mathrm{H}\right)$ \\
\hline
\end{tabular}

produced by this mechanism. Moreover, knowing the photon dose $I_{0}$ and $\sigma_{\mathrm{ph}-\mathrm{d}}$ values, it is possible to determine the half-life of a given molecule, as discussed by Bernstein et al. (2004).

In order to put our data on an absolute scale, after subtraction of a linear background and false coincidences coming from aborted double and triple ionization (see Simon et al. 1991), we have summed up the contributions of all cationic fragments detected and normalized them to the photoabsorption cross sections measured by Ishii \& Hitchcook (1988). Assuming a negligible fluorescence yield (due to the low carbon atomic number, Chen et al. 1981) and anionic fragments production in the present photon energy range, we assumed that all absorbed photons lead to cation formation.

The absolute cross section determination is described elsewhere (Papers I, II). Briefly, the non-dissociative single ionization (photoionization) cross-section $\sigma_{\mathrm{ph}-\mathrm{i}}$ and the dissociative single ionization (photodissociation) cross section $\sigma_{\mathrm{ph}-\mathrm{d}}$ of methanol can be determined by

$\sigma_{\mathrm{ph}-\mathrm{i}}=\sigma^{+} \frac{P I Y_{\mathrm{CH}_{3} \mathrm{OH}^{+}}}{100}$

and

$\sigma_{\mathrm{ph}-\mathrm{d}}=\sigma^{+}\left(1-\frac{P I Y_{\mathrm{CH}_{3} \mathrm{OH}^{+}}}{100}\right)$

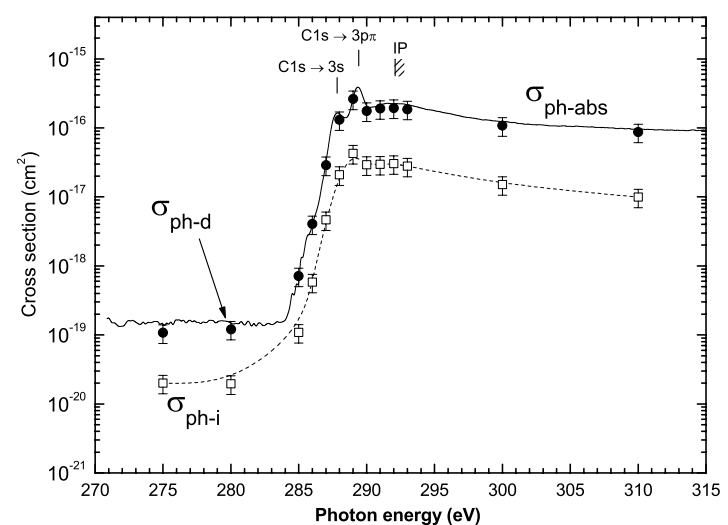

Fig. 4. Non-dissociative single ionization (photoionization) cross section, $\sigma_{\mathrm{ph}-\mathrm{i}}(\square)$, and dissociative ionization (photodissociation) crosssection, $\sigma_{\mathrm{ph}-\mathrm{d}}(\bullet)$, of methanol as a function of photon energy. The photoabsorption cross-section, $\sigma_{\text {ph-abs }}$ (solid line), taken from Ishii \& Hitchcook (1988) is also shown.

where $\sigma^{+}$is the cross section for single ionized fragments (see description in Papers I and II).

Both cross sections can be seen in Fig. 4 as a function of photon energy. The absolute absorption cross section of methanol (Ishii \& Hitchcook 1988) is also shown for comparison. Those values are also listed in Table 3. The estimated experimental error was about $30 \%$ which includes the data treatment, the uncertainties in the gas density in the interaction region and the loss of extremely energetic ions (initial kinetic energy $>30 \mathrm{eV}$ ).

\section{Summary and conclusions}

The aim of this work was to experimentally study the ionization and dissociation processes of the simplest interstellar alcohol, $\mathrm{CH}_{3} \mathrm{OH}$, employing soft X-ray photons $(100-310 \mathrm{eV})$ that go out from a toroidal grating monochromator (TGM) beamline of the Brazilian Synchrotron Light Laboratory (LNLS). The experimental set-up consists of a high vacuum chamber with a time-of-flight mass spectrometer (TOF-MS). Mass spectra were obtained using coincidence techniques.

Several ionic fragments produced by methanol photodissociation, have considerable kinetic energy (e.g. $\mathrm{H}^{+}, \mathrm{H}_{2}^{+}, \mathrm{C}^{+}$ and $\mathrm{O}^{+}$). An extension of this process to conditions in the 
Table 3. Values of non-dissociative single ionization (photoionization) cross section, $\sigma_{\mathrm{ph}-\mathrm{i}}$, and dissociative ionization (photodissociation) cross section, $\sigma_{\mathrm{ph}-\mathrm{d}}$, of $\mathrm{CH}_{3} \mathrm{OH}$ as a function of photon energy. The estimated experimental error was $30 \%$. The photoabsorption cross section $\left(\sigma_{\mathrm{ph}-\text { abs }}\right)$ from Ishii \& Hitchcook (1988) is also shown.

\begin{tabular}{lccc}
\hline \hline \multirow{2}{*}{$\begin{array}{l}\text { Photon } \\
\text { energy }(\mathrm{eV})\end{array}$} & \multicolumn{3}{c}{ Cross sections $\left(\mathrm{cm}^{2}\right)$} \\
\cline { 2 - 4 } 275 & $\sigma_{\mathrm{ph}-\mathrm{d}}$ & $\sigma_{\mathrm{ph}-\mathrm{i}}$ & $\sigma_{\mathrm{ph}-\mathrm{abs}}$ \\
\hline 280 & $1.1 \times 10^{-19}$ & $2.0 \times 10^{-20}$ & $1.3 \times 10^{-19}$ \\
285 & $1.2 \times 10^{-19}$ & $1.9 \times 10^{-20}$ & $1.4 \times 10^{-19}$ \\
286 & $7.1 \times 10^{-19}$ & $1.1 \times 10^{-19}$ & $8.2 \times 10^{-19}$ \\
287 & $4.1 \times 10^{-18}$ & $5.8 \times 10^{-19}$ & $4.6 \times 10^{-18}$ \\
288 & $2.9 \times 10^{-17}$ & $4.6 \times 10^{-18}$ & $3.4 \times 10^{-17}$ \\
289 & $1.3 \times 10^{-16}$ & $2.1 \times 10^{-17}$ & $1.5 \times 10^{-16}$ \\
290 & $2.6 \times 10^{-16}$ & $4.3 \times 10^{-17}$ & $3.1 \times 10^{-16}$ \\
291 & $1.8 \times 10^{-16}$ & $2.9 \times 10^{-17}$ & $2.1 \times 10^{-16}$ \\
292 & $1.9 \times 10^{-16}$ & $2.9 \times 10^{-17}$ & $2.2 \times 10^{-16}$ \\
293 & $1.9 \times 10^{-16}$ & $3.0 \times 10^{-17}$ & $2.1 \times 10^{-16}$ \\
300 & $1.9 \times 10^{-16}$ & $2.8 \times 10^{-17}$ & $2.2 \times 10^{-16}$ \\
310 & $1.1 \times 10^{-16}$ & $1.5 \times 10^{-17}$ & $1.2 \times 10^{-16}$ \\
& $8.7 \times 10^{-17}$ & $9.9 \times 10^{-18}$ & $9.7 \times 10^{-17}$ \\
\hline
\end{tabular}

interstellar medium suggests endothermic ion-molecule (or radical-molecule) reactions may become important in elucidating the pathways of formation of some complex molecules (Largo et al. 2004). Unlike previous work with formic acid (Paper I) performed in the same spectral range, no fragments with large kinetic energy fragments have been observed due the single photoionization of $\mathrm{CH}_{3} \mathrm{OH}$.

We have found that about $11-16 \%$ of $\mathrm{CH}_{3} \mathrm{OH}$ survives soft X-ray ionization field. $\mathrm{COH}^{+}\left(\right.$or $\mathrm{HCO}^{+}$), $\mathrm{H}^{+}$and $\mathrm{CH}_{2} \mathrm{OH}^{+}$were the main fragments produced by high energy photons. This large resistance to the X-ray photons could help to sustain the large column density observed for methanol in star-forming regions.

Dissociative and non-dissociative photoionization cross sections in the energy range of $275 \mathrm{eV}$ to $310 \mathrm{eV}$ (over the $\mathrm{C} 1 \mathrm{~s}$ edge) were also determined. We hope that the molecular cross sections derived in this work will give rise to more precise values for some molecular abundances in interstellar medium chemistry models.

Acknowledgements. The authors would like to thank the staff of the Brazilian Synchrotron Facility (LNLS) for their valuable help during the course of the experiments. We are particularly grateful to Dr. R. L. Cavasso and Professor A. N. de Brito for the use of the Time-of-Flight Mass Spectrometer. This work was supported by LNLS, CNPq and FAPERJ.

\section{References}

Allamandola, L. J., Sandford, S. A., \& Valero, G. J. 1988, Icarus, 76225

Azuma, Y., Mishima, Y., Senba, Y., Yoshida, H., \& Hiraya, A. 2005, J. Elect. Spectr. Relat. Phenom., 144, 183

Bernstein, M. P., Ashbourn, S. F. M., Sandford, S. A., \& Allamandola, L. J. 2004, ApJ, 601, 365

Bockelee-Morvan, D., Crovisier, J., Colom, P., \& Despois, D. 1994, A\&A, 282, 647

Burton, G. R., Chan, W. F., Cooper, G., \& Brion, C. E. 1992, Chem. Phys., 167, 349

Boechat-Roberty, H. M., Pilling, S., \& Santos, A. C. F. 2005, A\&A, 438, 915 (Paper I)
Caswell, J. L., Vaile, R. A., Ellingsen, S. P., Whiteoak, J. B., \& Norris, R. P. 1995, MNRAS, 272, 96

Caswell, J. L., Gardner, F. F., Norris, R. P., et al. 1993, MNRAS, 260, 425

Charnley, S. B., Kress, M. E., Tielens, A. G. G. M., \& Millar, T. J. 1995, ApJ, 448,232

Charnley, S. B., Tielens, A. G. G. M., \& Rodgers, S. D. 1997, ApJ, 482, L203

Chen, M. H., Crasemann, B., \& Mark, H. 1981, Phys. Rev. A, 24, 177

Combes, F., Gerin, M., Wooten, A. S., et al. 1987, A\&A, 180, L13

Crovisier, J., \& Bockelée-Morvan, D. 1999, Space Sci. Rev., 90, 19

De Buizer, J. M., Piña, R. K., \& Telesco, C. M. 2000, ApJS, 130, 437

Goldsmith, P. F., Langer, W. D., \& Velusamy, T. 1999, A\&AS, 194, 681

Goicoechea, J. R., Rodriguez-Fernandez, N. J., \& Cernicharo, J. 2004, ApJ, 600, 214

Grim, R. J. A., Baas, F., Greenberg, J. M., Geballe, T. R., \& Schutte, W. 1991, A\&A, 243, 473

Hansen, D. L., et al. 1998, Phys. Rev. A, 58, 5

Herbst, E., \& Leung, C. M. 1986, MNRAS, 222, 689

Hudson, R. L., \& Moore, M. H. 1999, Icarus 140, 451

Irvine, W. M., Goldsmith, P. F., \& Hjalmarson, A. 1987, in Interestelar Processes, ed. D. J. Hollenbach, \& H. A. Thronson, Jr. (Dordrech: Reidel)

Ishii, I., \& Hitchcook, A. P. 1988, J. Elect. Spectr. Relat. Phenom., 46, 55

Largo, A., Redondo, P., \& Barrientos, C. 2004, Int. J. Quant. Chem., 98, 355

Lee, H.-H., Bettens, R. P. A., \& Herbst, E. 1996, A\&AS, 119, 111

Keane, J. V., Tielens, A. G. G. M., Boogert, A. C. A., Schutte, W. A., \& Whittet, D. C. B. 2001, A\&A, 376, 254

Menten, K. M. 1991, ApJ, 380, L75

Millar, T. J., Herbst, E., \& Charnley, S. B. 1991, ApJ, 369, 147

Moore, M. H., \& Hudson, R. L. 1998, Icarus, 135, 518

Mumma, M. J., Dello, Russo N., DiSanti, M. A., et al. 2001, Science, 292, 1334

Nenner, I., \& Morin, P. 1996, VUV and Soft X-Ray Photoinization, ed. Uwe Becker, \& D. A. Shirley (New York: Plenum Press)

Norris, R. P., Whiteoak, J. B., Caswell, J. L., Wieringa, M. H., \& Gough, R. G. 1993, ApJ, 412, 222

Pei, C. C., Liu, S.-Y., \& Snyder, L. E. 2000, ApJ, 530, 800

Pilling, S., Boechat-Roberty, H. M., \& Santos, A. C. F. 2006, A\&A, 449, 1289 (Paper II)

Pontoppidan, K. M., Fraser, H., Dartois, E., et al. 2003, A\&A, 408, 981

Pontoppidan, K. M., van Dishoeck, E. F., \& Dartois, E. 2004, A\&A, 426, 925

Prince, K. C., Richter, R., Simone, M., Alagia, M., \& Coreno, M. 2003, J. Phys. Chem. A, 107, 1955

Remijan, A. J., Shiao, Y.-S., Friedel, D. N., Meier, D. S., \& Snyder, L. E. 2004, ApJ, 617, 384

Requena-Torres, M. A., Martín-Pintado, J., Rodríguez-Franco, A., et al. 2006, A\&A in press

Santos, A. C. F., Lucas, C. A., \& de Souza, G. G. B. 2001, J. Elect. Spectr. Relat. Phenom., 114, 115

Schutte, A. J., van der Walt, D. J., Gaylard, M. J., \& MacLeod, G. C. 1993, MNRAS, 261, 783

Schutte, W. A., Gerakines, P. A., Geballe, T. R., van Dishoeck, E. F., \& Greenberg, J. M., 1996, A\&A, 309, 633

Shalabiea, O. M., \& Greenberg, J. M. 1994, A\&A, 290, 266

Shi, Y. J., Consta, S., Das, A. K., et al. 2002, J. Chem. Phys., 116, 6990

Slysh, V. I., Valtts, I. E., Kalenskii, S. V., et al. 1999, A\&AS, 134, 115

Stolte, W. C., Ohrwall, G., Sant'Anna, M. M., et al. 2002, J. Phys. B: At. Mol. Opt. Phys., 35, L253

Szymczak, M., Hrynek, G., \& Kus, A. J. 2000, A\&AS, 143, 269

Simon, M., LeBrun, T., Morin, P., Lavolée, M., \& Maréchal, J. L. 1991, Nucl. Instrum. Methods B, 62, 167

Sorrell, W. H. 2001, ApJ, 555, L129

Tang, X. P., Gao, L. R., Wang, Y. L., et al. 2002, Chinese Science Bulletin, 47, 1973

Tielens, A. G. G. M., \& Allamandola, L. J. 1987, in Interestellar Processes, ed. D. J. Hollenbach, \& H. A. Thronson (Dordrech: Reidel), 397

Tielens, A. G. G. M., \& Whittet, D. C. B. 1997, IAUS, 178, 45

Watanabe, N., \& Kouchi, A. 2002, ApJ, 571, L173

Walsh, A. J., Hyland, A. R., Robinson, G., \& Burton, M. G. 1997, MNRAS, 291, 261

Wiley, W. E., \& McLaren, I. W. 1955, Rev. Sci. Instrum., 26, 1150

Woon, D. E. 2002, ApJ, 571, L177

Zavilopulo, A. N., Chipev, F. F., \& Kokhtych, L. M. 2005, Nucl. Instrum. Meth. Phys. Res. B, 233, 302 